\title{
Antenatal variant of Bartter syndrome
}

\author{
S S Vithanage Sukumar ${ }^{1}$, P M G Punchihewa ${ }^{2}$ \\ Sri Lanka Journal of Child Health, 2009; 38: 138-139
}

(Key words: Bartter syndrome, antenatal variant)

\section{Introduction}

Bartter and Gitelman syndromes are renal salt wasting disorders in which the kidneys cannot reabsorb chloride in the thick ascending limb of the loop of Henle (TALH) or the distal convoluted tubule (DCT), depending on the mutation ${ }^{1}$. Bartter syndrome was first described in 1962 by Fredric Bartter $^{2}$, who observed the association of hyperplasia of juxtaglomerular complex with hyperaldosteronism and hypokalaemic metabolic alkalosis.

The antenatal variant of Bartter Syndrome is the most severe of all variants and has both the features of metabolic alkalosis (from the low potassium) as well as profound systemic manifestations ${ }^{1}$. It is characterized by polyhydramnios due to polyuria in utero which is complicated by premature delivery ${ }^{1}$. After birth, life threatening episodes of fever and dehydration occur secondary to profound polyuria, vomiting and diarrhoea. Severe electrolyte imbalances and marked growth retardation are typical $^{1}$. Dysmorphic features, including triangular facies, prominent forehead, protruding ears, large eyes with strabismus and drooping mouth, may be present $^{3}$. The majority of these infants also suffer from severe hypercalciuria with associated nephrocalcinosis and osteopenia. These infants also have an abnormally high level of prostaglandin E2 production ${ }^{1}$. A subset of patients with antenatal Bartter syndrome develops sensorineural deafness, which is detectable with audiometry ${ }^{1}$.

\section{Case report}

A 5 month old baby boy, the third child of nonconsanguineous parents with 2 elder normal children, was transferred to our ward from the premature baby clinic at Castle Street Hospital for Women (CSHW)

${ }^{1}$ Senior Registrar in Paediatrics, ${ }^{2}$ Consultant Paediatrician, Lady Ridgeway Hospital, Colombo.

(Received on 22 September 2008. Accepted on 20 October 2008) for investigation of failure to thrive. He was delivered at 33 weeks of gestation by elective lower segment caesarean section (LSCS) due to severe degree of polyhydramnios. The antenatal period was complicated by polyhydramnios from 25 weeks of gestation and amniocentesis was done at 31 weeks due to respiratory distress in the mother. At birth the weight was $2.29 \mathrm{~kg}$ and the maturity was 34 weeks but there was no birth asphyxia. She was kept in the premature baby unit (PBU) and was treated for suspected sepsis with intravenous antibiotics for 12 days due to reduced activity and jaundice. However, blood culture showed no growth and lumbar puncture was not done. After discharge from the PBU with a weight of $2.2 \mathrm{~kg}$ he was exclusively breast fed and there were no vomiting or feeding difficulties. However, the baby had severe failure to thrive and was investigated at the baby clinic at CSHW. There were no recurrent infections or diarrhoeal illness and mother said there was no polyuria. The ultrasound scans (USS) of abdomen and brain and the $2 \mathrm{D} \mathrm{ECHO}$ were normal.

The baby had a lower respiratory tract infection on admission to the Lady Ridgeway Hospital (LRH) and also moderate to severe dehydration with no significant diarrhoea or vomiting. Urine output was not reduced despite the dehydration. On admission, the blood urea was $11.2(1.5-3) \mathrm{mmol} / \mathrm{L}$, serum creatinine $31(<44)$ micromol/L, serum sodium 136 and potassium $2.4 \mathrm{mmol} / \mathrm{L}$. It was difficult to correct hydration and electrolytes despite oral and intravenous replacement. The venous blood gas showed a metabolic alkalosis with a $\mathrm{pH}$ of 7.5, a base excess of 19.5 and a bicarbonate of 42.9 . The serum osmolality was $279(275-295) \mathrm{mosmol} / \mathrm{kg}$ water and the urine osmolality $212(50-1200) \mathrm{mosmol} / \mathrm{kg}$ water. The U/S osmolality ratio was $0.76(>2.0)$ despite dehydration. The urinary electrolytes showed sodium of $53(<20) \mathrm{mmol} / \mathrm{L}$, potassium of $28.1 \quad(<20)$ $\mathrm{mmol} / \mathrm{L}$ and chloride of $63.0 \mathrm{mmol} / \mathrm{L}$ confirming excessive loss of urinary electrolytes. The USS of the abdomen showed bilateral medullary nephrocalcinosis. 
A diagnosis of the antenatal variant of Bartter syndrome was made and treatment was started with indomethacin, a prostaglandin inhibitor. The hypokalaemia and alkalosis did not fully resolve with this treatment. The hydration was maintained with normal maintenance of fluids. The urine calcium: creatinine ratio was 0.09 which is within the normal range but this was done while on treatment. Spironolactone was added as electrolyte and acid base balance was not fully corrected with indomethacin and oral potassium supplements.

\section{Discussion}

In the antenatal Bartter syndrome an autosomal recessive mode of inheritance is observed in some patients, although many cases are sporadic ${ }^{4}$. In this form of Bartter syndrome 2 genotypes were previously identified as Type I which results from mutations in the sodium-potassium-2 chloride cotransporter (the site of action of frusemide) gene (NKCC gene) and Type II which result from mutations in the apical ATP-regulated potassium channel (ROMK gene) ${ }^{4}$. Recent studies have identified two novel types IV and V associated with sensorineural deafness. Type IV is caused by mutations in the BSND gene which encodes barttin, an essential subunit that is required for the trafficking of the chloride channel, CLC-K (both subunits $\mathrm{ClC}$ $\mathrm{Ka}$ and $\mathrm{ClC}-\mathrm{Kb}$ ) to the plasma membrane and Type $\mathrm{V}$ is caused by mutations in the genes encoding the subunits of chloride channel CLC-K ${ }^{4}$. The primary pathogenic mechanism in antenatal Bartter syndrome involves defective transepithelial chloride reabsorption in the $\mathrm{TALH}^{4}$. Failure to reabsorb chloride results in a failure to reabsorb sodium and leads to excessive sodium and chloride delivery to the distal tubules, leading to excessive salt and water loss from the body. Other pathophysiological abnormalities result from excessive salt and water loss. The renin-angiotensin-aldosterone system (RAAS) is a feedback system activated with volume depletion. Long term stimulation may lead to hyperplasia of the juxtaglomerular complex ${ }^{4}$.

Angiotensin II is directly vasoconstrictive, increasing both systemic and renal arteriolar constriction, which helps prevent systemic hypotension. It directly increases proximal tubular sodium reabsorption. Angiotensin II induced renal vasoconstriction, along with potassium deficiency, produces a counterregulatory rise in vasodilating prostaglandin $\mathrm{E}$ (PGE) levels ${ }^{4}$. High PGE levels are associated with growth inhibition in children.
Excessive distal sodium delivery increases distal tubular sodium reabsorption and exchange with the electrically equivalent potassium or hydrogen ion. This in turn promotes hypokalaemia, while lack of chloride reabsorption promotes inadequate exchange of bicarbonate for chloride, and the combined hypokalaemia and excessive bicarbonate retention lead to metabolic alkalosis.

Normally, reabsorption of the negative chloride ions promotes a lumen-positive voltage, driving positive calcium and magnesium absorption through paracellular channels. Dysfunction of the TALH chloride transporters prevents urine calcium reabsorption. Hypercalciuria leads to nephrocalcinosis.

Treatment of Bartter syndrome is directed at preventing dehydration, maintaining nutritional status and correcting hypokalaemia ${ }^{3}$. Even with appropriate therapy, serum potassium values may not normalize particularly in patients with the neonatal form and this was seen in our patient. Cyclooxygenase inhibitors, e.g. indomethacin, have been shown to reverse all the clinical and laboratory derangements, save for those related to calcium.

With close attention to electrolyte balance, volume status and growth, the long term prognosis is generally good. However in a small minority of patients, hyperoxaluria, nephrocalcinosis and chronic indomethacin therapy can lead to chronic interstitial nephritis and chronic renal failure ${ }^{3}$.

\section{References}

1. Frassetto LA. Bartter Syndrome. eMedicine May 2008. Available from: http://www.emedicine.com/med/TOPIC213.HTM

2. Bartter FC, Pronove P, Gill JR. Hyperplasia of the juxtaglomerular complex with hyperaldosteronism and hypokalemic alkalosis. American Journal of Medicine. 1962; 33:811-28.

3. Behrman RE, Kliegman RM, Jenson HB, editors, Nelson Text Book of Paediatrics $17^{\text {th }}$ ed. Philadelphia: W B Saunders; 2004. p. 1763.

4. Devarajan P. Bartter Syndrome. e-Medicine August 2006. Available from: http://www.emedicine.com/PED/topic210.htm 